Musées, Patrimoine et Culture scientifiques et techniques

$175 \mid 2018$

janvier-février 2018

\title{
Des visiteurs émancipés : lycéens et étudiants filment au musée
}

Isabelle Roussel-Gillet

\section{OpenEdition \\ Journals}

Édition électronique

URL : http://journals.openedition.org/ocim/1892

DOI : $10.4000 /$ ocim. 1892

ISSN : 2108-646X

Éditeur

OCIM

Édition imprimée

Date de publication : 1 janvier 2018

Pagination : 12-15

ISSN : 0994-1908

Référence électronique

Isabelle Roussel-Gillet, «Des visiteurs émancipés : lycéens et étudiants filment au musée », La Lettre de I'OCIM [En ligne], 175 | 2018, mis en ligne le 01 janvier 2019, consulté le 01 mai 2019. URL : http:// journals.openedition.org/ocim/1892 ; DOI : 10.4000/ocim.1892

Ce document a été généré automatiquement le 1 mai 2019.

Tous droits réservés 


\title{
Des visiteurs émancipés : lycéens et étudiants filment au musée
}

\author{
Isabelle Roussel-Gillet
}

Tournage de Buller au musée dans l'exposition Versailles au musée des Beaux-Arts d'Arras 2015.

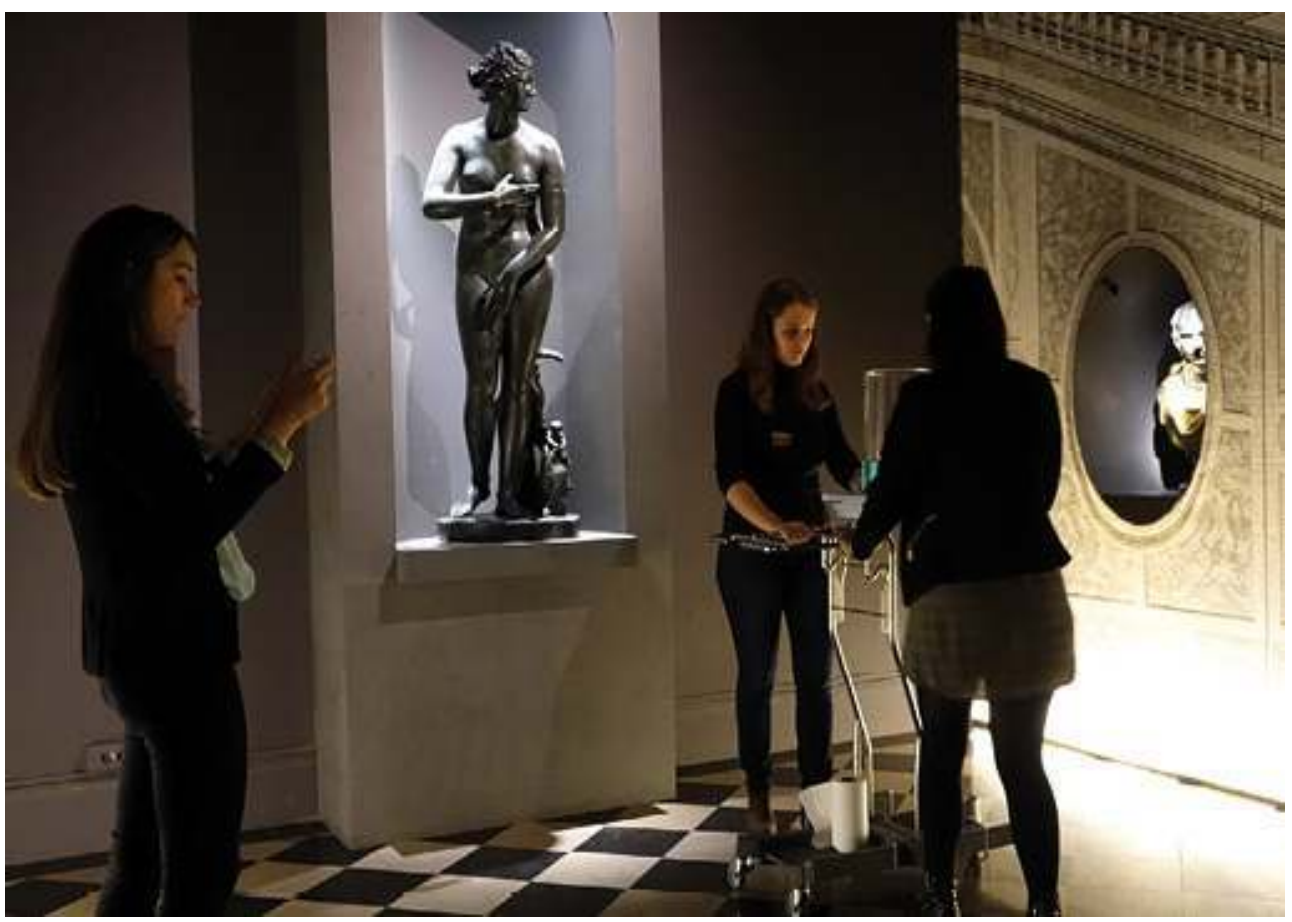

(C) L'Art de muser

1 La création du festival "Musées(em)portables" en 2012 est venue "d'une observation qui relie le public au téléphone portable, mode "global" de communication, passeport universel vers l'autre", pouvons-nous lire sur le site des organisateurs (1). Faire un film de trois minutes maximum avec son téléphone portable et ainsi devenir réalisateur/auteur et/ou acteur au musée, sans budget : telle est la proposition de museumexperts (2). Elle permet 
d'inventer un nouveau rapport décomplexé à l'institution muséale. Fondamentalement, l'opération atteste des transformations en cours entre les institutions muséales et les citoyens qui inventent de nouveaux usages. Pour sa sixième édition, en 2017, 96 films ont concouru, dont 51 venus des Hauts-de-France, réalisés tant par les étudiants du master que par les lycéens. Ces 51 films impulsés et visionnés dans le cadre des actions culturelles du master expographie-muséographie de l'université d'Artois nous donnent l'occasion de croiser trois points de vue : celui du musée, du référent encadrant et des jeunes réalisateurs. Il est bien question de passeport vers une altérité, chacun des trois acteurs se découvre grâce à la relation avec les deux autres.

\section{L'opération "Musées(em)portables" en Hauts-de- France}

Constatant l'absence de films en provenance des Hauts-de-France au concours du Sitem lors des quatre premières éditions, et convaincu de l'aspect heuristique de l'opération, le master expographie-muséographie a impulsé pour les éditions de 2016, 2017 et 2018 l'idée de parrainage entre un lieu d'exposition de cette région et un groupe de jeunes pour la production de films. Ces jeunes réalisateurs, souvent par équipe de 3 à 5 , sont tantôt issus de la même classe de lycée ou d'une option, tantôt réunis par simple affinité sur temps périscolaire. En complète cohérence avec l'esprit de la formation en muséographie, qui est d'abord pratique et partenariale, mais aussi itinérante dans les sites, les masteriens sont invités à expérimenter eux-mêmes, puis à développer leur réseau en coordonnant la mise en place d'une action culturelle. Par équipe, il s'agit d'abord pour eux de produire un film et de se prêter ainsi à l'exercice. Pour cela un workshop de trois jours, encadré par l'artiste vidéaste Benoît Labourdette, se déroule au musée des Beaux-Arts d'Arras. Une réunion de lancement des jumelages avec les musées de la région a lieu au terme du workshop avec présentation du travail réalisé. Puis l'automne est dédié à la conduite des jumelages et à la production des films par les groupes de jeunes réalisateurs d'une quinzaine d'établissements. Ces films, que ce soient les tout premiers réalisés par les étudiants du master et ceux réalisés par les lycéens dans le cadre des jumelages sont envoyés pour concourir, mais aussi présentés lors d'une séance publique en décembre à la Maison étudiante de l'université. C'est alors l'occasion de réunir ces jeunes collégiens ou lycéens venant souvent pour la première fois à l'université, leurs encadrants, les responsables et représentants des musées et les masteriens du master expographiemuséographie. Ce temps fort est un moment de convivialité pour insister sur la dimension ludique et volontaire de l'opération, sur le décloisonnement des lieux de formation. Il s'agit bien sûr aussi de mettre en avant le musée comme lieu propice à l'imaginaire, à la fiction, tout en valorisant les capacités à la scénarisation et à l'écriture. L'implication du master découle également de la conviction que la maîtrise de la production d'images est devenue essentielle dans les compétences métier. 


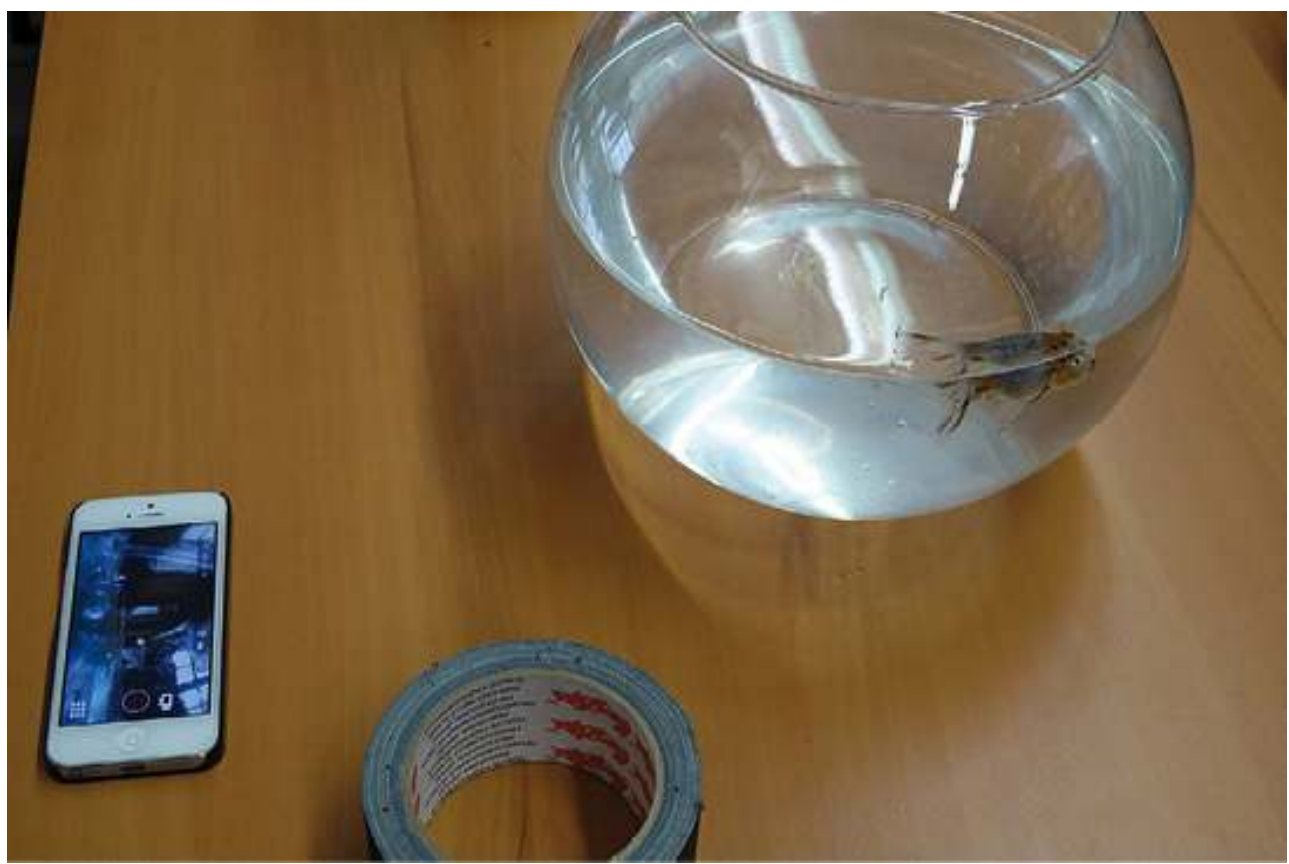

(c) L'Art de muser

Écrire un storyboard.

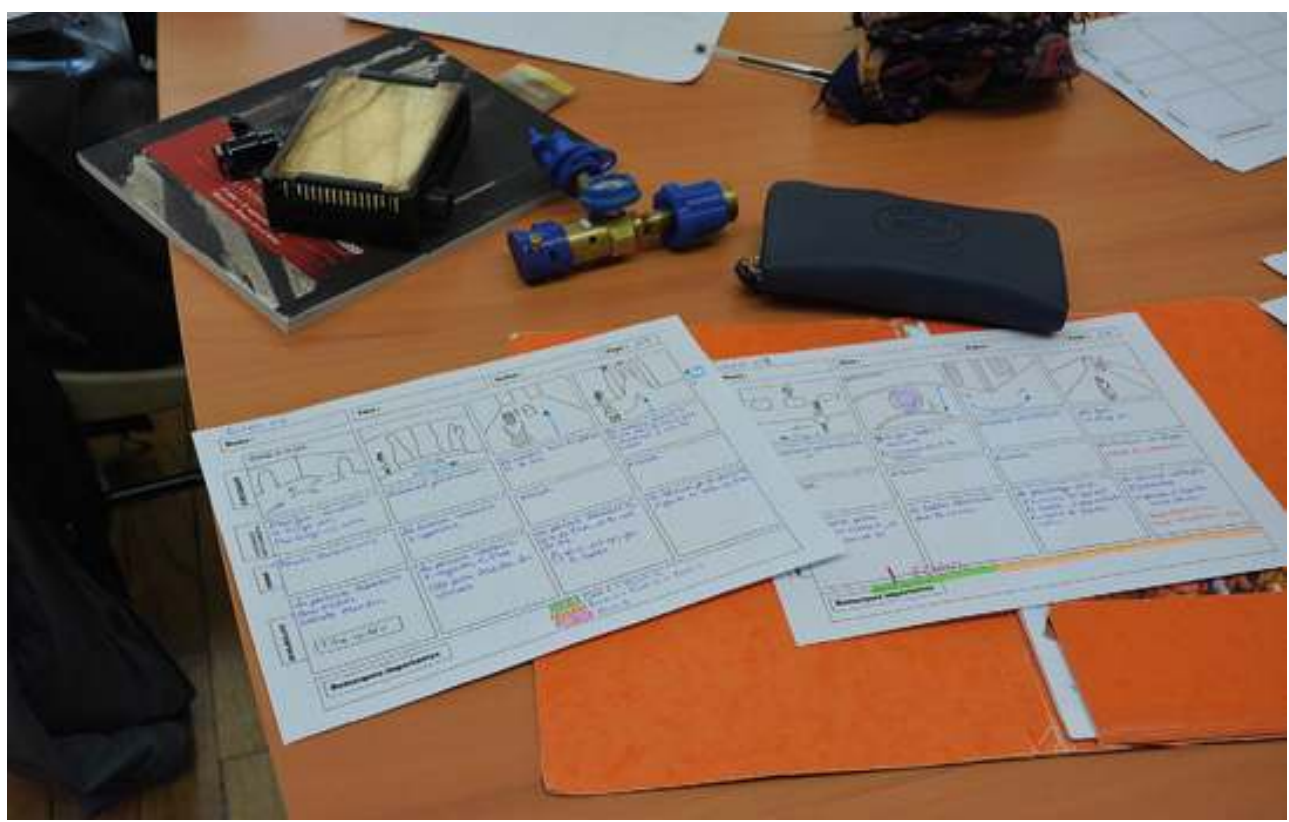

(c) L'Art de muser

\section{"Musées(em)portables", pour une visitaction}

Dispositif léger en main, le jeune réalisateur, qu'il soit lycéen ou étudiant, ne s'apprête pas à faire un film documentaire sur le musée, ou un film de communication pour le musée, il ose une fiction, pose un regard sur le lieu, ses objets, saisit une atmosphère, 
invente sa propre histoire. Il adopte une liberté de ton ou de genre. De visiteur passif ou captif du cadre scolaire, il devient acteur. À la place d'une visite guidée didactique, un accueil encourageant rappelle simplement quelques règles de respect vis-à-vis des autres visiteurs du jour. La mise en situation de devoir produire donne un cadre clair et non attentiste. Ce n'est néanmoins pas le résultat qui est "jugé", mais le chemin qui est facilité. Rares sont ceux qui se vouent à devenir des professionnels de l'audiovisuel.

4 L'intérêt majeur pour le musée est de nouer un lien avec un public qualifié de peu réceptif ou de très volatile : l'adolescent et jeune adulte, de 14 à 19 ans. Public dont on sait l'audience traditionnellement difficile à capter. Dès lors que le téléphone portable n'est plus perçu comme un perturbateur d'une attention réclamée mais un outil allié, les potentialités techniques et les usages novateurs peuvent être mobilisés. Possibilité de montage, recours à des applications spécifiques ou spontanéité de l'appropriation. Le rapport au téléphone souvent perçu comme prothétique n'est plus jugé, puisqu'il s'agit d'un rapport naturel mobilisé comme une ressource parmi d'autres. La charte Tous photographes est pour beaucoup dans la possibilité acquise de désormais filmer les œuvres de l'exposition permanente en toute liberté, en France (3).

Mélanie Lerat, directrice adjointe du musée des Beaux-Arts d'Arras, qui a accueilli des jeunes de lycée général comme professionnel et de master lors de deux éditions de "Musées(em)portables" témoigne : "Décalage, humour, impertinence, souvent sans détour. Si accueillir une équipe concourant à Musées(em)portables n'est pas une sinécure pour le musée des Beaux-Arts d'Arras - filmer dans un musée ? Danser, courir, chanter? Faire parler un cartel tombé à terre, un socle vide? -, c'est avant tout la formidable opportunité de porter un regard nouveau sur une collection et un bâtiment d'exception. Habité par cette dynamique, le musée descend ainsi de son piédestal : il devient un tremplin pour l'imagination. La curiosité de l'équipe du musée lors du visionnage des films réalisés, tout comme le succès rencontré par la projection des films lors de la Nuit des musées, témoignent de ce plaisir de la réinvention, toujours surprenante.

Cela passe aussi par la (ré)appropriation des codes et des attitudes propres aux musées, vivre le lieu autrement pour celui qui filme, celui qui joue, celui qui accueille et enfin celui qui regarde. Le dispositif Musées(em)portables infléchit l'image que l'on a du musée, au sein même des équipes qui $y$ travaillent quotidiennement.

$7 \quad A u$ fil des éditions et des collaborations avec le master expographie-muséographie, cette opération se révèle aussi être un moteur efficace pour fédérer un territoire : elle relie les étudiants de l'université d'Artois à leur musée "de proximité" et aux structures éducatives du territoire, puis ces dernières au musée des Beaux-Arts d'Arras. Enfin, lors de la prochaine édition, les étudiants travailleront avec une structure sociale, partenaire régulier des actions du musée, l'épicerie solidaire Le Petit Marché Arrageois". 
Tournage de Merci Simon au musée des Beaux-Arts d'Arras

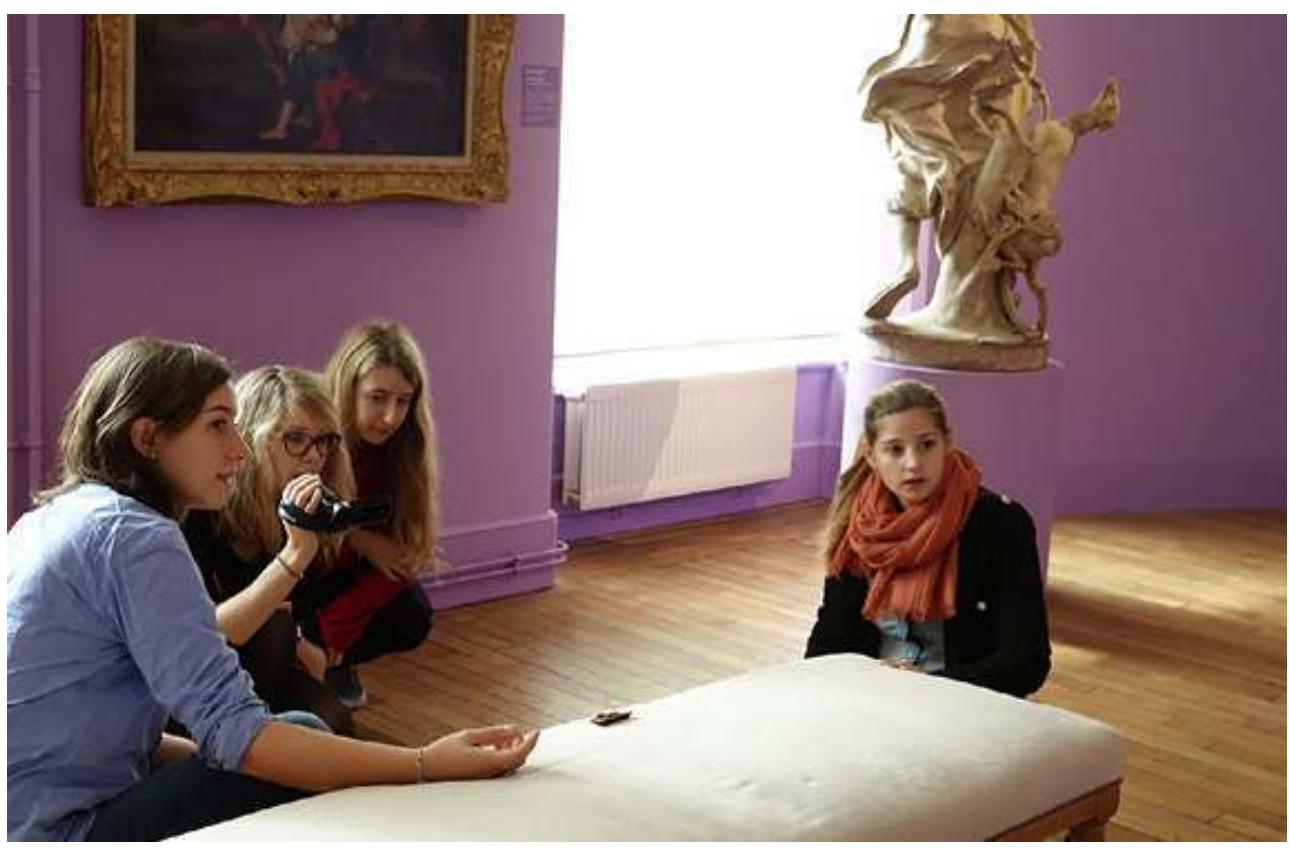

(c) L'Art de muser

\section{L'accompagnant du projet, un facilitateur}

Stéphanie Jovenel, professeur de Lettres au lycée Saint-Paul de Lille, explique comment elle intègre cette réalisation de film dans son programme : "Pour la seconde année consécutive, le projet Musées(em)portables a été mené avec les élèves de Terminale qui présentent l'option facultative Histoire des Arts au baccalauréat. Le projet s'inscrit d'autant plus dans leur formation que l'un des thèmes du baccalauréat porte depuis 2016 sur la scénographie de l'art. Tous les élèves de l'option ont souhaité y participer, motivés à l'idée de s'approprier l'espace muséal de façon moins conventionnelle qu'en tant que simples visiteurs. Les quatorze jeunes, répartis en trois groupes, ont ainsi réalisé trois films en partenariat avec le Palais des Beaux-Arts de Lille. Trois séances au musée, dont une avec une étudiante de master expographie-muséographie, ont été nécessaires. En alternance avec ces séances, chaque groupe, guidé par l'enseignant, a consacré en classe des temps de réflexion au choix des scénarii ainsi qu'à la faisabilité de leur mise en cuvre. De façon créative, un groupe a eu l'idée d'utiliser leur avatar Sim's, un autre a construit son scenario sur fond de jeu vidéo, le dernier a filmé grâce à une voiture télécommandée... La proximité du Palais des Beaux-arts de Lille avec le lycée a facilité le processus de réalisation; les élèves qui l'ont souhaité ont pu s'y rendre par leurs propres moyens et sur leur temps libre. Le projet Musées (em)portables s'inscrit aussi dans le projet pédagogique ; les films réalisés ont été visionnés en classe et évalués selon des critères préalablement définis en commun avec les élèves : l'originalité du scénario, la bande-son ou la qualité du montage, l'investissement de chacun. Musées (em)portables est un précieux allié pour l'enseignant et a fortiori pour l'enseignant d'histoire des arts, il est un média qui "emporte" les élèves vers une (re)découverte du musée qui devient ainsi plus humain, plus vivant et devient un lieu d'enrichissement que le lycéen s'approprie plus volontiers." 
9 Pour les étudiants du master expographie-muséographie un autre enjeu est de penser ces films comme une véritable action culturelle. Ils sont à l'interface entre deux mondes professionnels, celui des musées et celui des structures éducatives, sociales, ou d'animation... Ce qui leur a permis de travailler tant avec des adultes accompagnés qu'avec des publics moins coutumiers, comme des bénéficiaires du RSA. C'est dire que la démarche est transférable avec d'autres publics. Pour cette présente édition, des jeunes de troisième au collège jusqu'aux terminales de lycée ont participé. Au questionnaire anonyme qui leur a été proposé ils répondent que c'est un projet "qui change". L'analyse qualitative, quant à elle, fait apparaître des bénéfices pour des jeunes "décrocheurs". Le film de témoignages de la série "Médiation singulière" tourné par Juliette Gouesnard donne la parole à deux garçons qui s'ennuient en classe et ont pourtant mené au bout la réalisation d'un film. Un autre témoignage audiovisuel recueilli par Camille Roussel reflète une liberté de ton et une aisance avec l'outil, c'est ainsi que la jeune fille a choisi de répondre aux questions depuis sa chambre et par webcam (4). Cette porosité entre la sphère familiale, publique du musée et éducative n'est pas le moindre intérêt puisqu'il permet un continuum susceptible de briser les clivages, de désacraliser le musée.

\section{Panorama des productions, ce que les jeunes filment du musée}

10 Le visionnage des 51 films produits cette année nous permet de dégager quelques tendances : l'exploration de musées variés, de société, d'histoire naturelle, d'arts et de traditions populaires, même si le musée des beaux-arts est massivement choisi. Le film opte pour des tons bien tranchés : l'humour de la parodie d'un genre, la poésie pour saisir une atmosphère, le récit dramatique de la reconstitution docu-fiction (de la vie des mineurs par exemple), ou l'ancrage dans l'actualité (le thème des migrants). L'arrêt sur une œuvre prédomine sur le regard sur le bâti, qui est davantage prégnant lorsque le lieu est patrimonial. La culture personnelle du jeune est facilement convoquée pour faire référence à des séries, des films ou des jeux vidéo, ce qui favorise les generation joke. Le jeune active ses ressources instrumentales tout comme sa culture. Sensibles à la rencontre des cultures, nous pouvons nous poser la question de savoir qui a à apprendre quoi de qui ? Le fait que soient convoqués des avatars en référence à un jeu vidéo tend à légitimer la culture pratiquée par ces jeunes sans hiérarchisation par rapport à un lieu vécu comme élitiste. Introduire ces nouvelles technologies au musée contraste fortement avec une autre tendance dominante : celle de baigner dans la nostalgie d'un rapport au passé, à la mémoire.

11 Autre tendance : le jeune réalisateur met souvent l'institution en mouvement, il y danse, court ; il en fait au sens propre un terrain de jeu. Liberté de corps et de ton sont au rendez-vous pour transgresser certains codes. Ce qui nous est dit du musée renvoie alors aux interdits, comme le "ne pas courir", ou au fantasme du vol. Enfin nous retiendrons les films qui donnent la parole aux objets, aux personnages, rompant ainsi le silence du lieu. Ces interprétations plurielles manifestent une compréhension sensible et un regard sociologique sur des codes fondés sur le silence, la lenteur, l'arrêt ou la distance, qui caractérisent une image assimilée de la visite au musée des Beaux-Arts. 


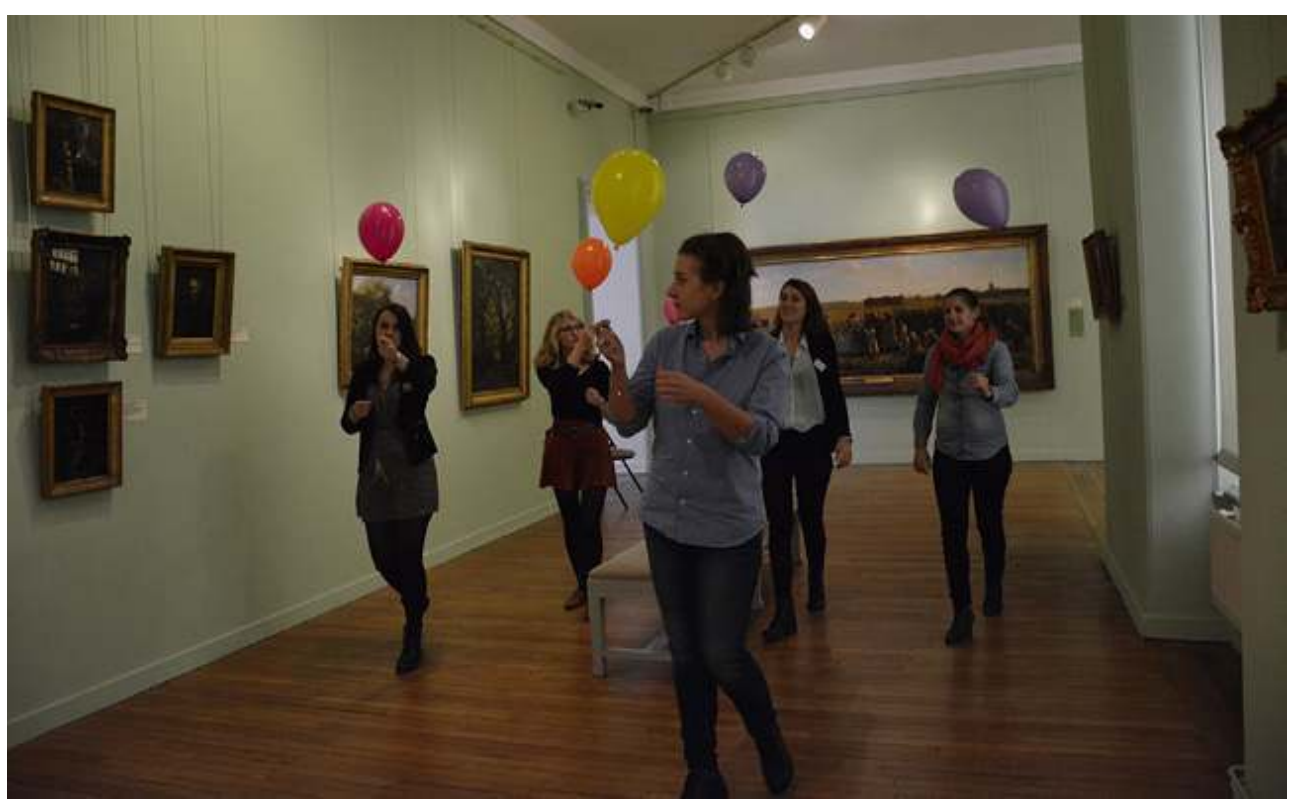

(c) L'Art de muser

\section{Alors, des films pour s'émanciper de quoi ?}

Peut-être s'agit-il d'émanciper le jeune visiteur de cette vision très codée pour l'ouvrir sur une vision plurielle des musées et sur d'autres possibles. Sur le court terme, l'action est émancipatrice. Une modification à moyen ou long terme nécessiterait une convergence d'autres actions culturelles. Ce qui nous intéresse particulièrement c'est le double changement de statut, nous l'avons évoqué, celui du musée et celui de l'action éducative. D'une part, le musée a l'occasion de dépasser son statut de lieu de visite pédagogique et de se découvrir en terrain d'expérimentation familier et accueillant, et d'autre part, les enseignants saisissent l'occasion de dépasser le caractère formel et scolaire pour explorer des approches d'éducation informelle. Très en phase avec l'esprit de l'éducation populaire qui réconcilie l'acculturation et la créativité, l'expression de soi et la découverte de l'autre (le travail en équipe, le sens du projet et du collectif, la coconstruction...), il s'agit bien d'émancipation au sens fort du mot. C'est aussi pour cette raison que le public concerné peut être à tous les âges de la vie (pourquoi pas demain "Musées(em)portables" avec une maison de retraite ?), et de toutes les conditions sociales (travailler avec des centres de rééducation...).

13 La ministre de la Culture Audrey Azoulay ne s'y était pas trompée : elle avait effectivement repéré dans les conclusions et propositions du rapport sur les musées du $\mathrm{XXI}^{\mathrm{e}}$ siècle l'action "Musées(em)portables". Elle a demandé, avant son départ du ministère, dans une lettre de cadrage aux directions régionales des affaires culturelles de soutenir et encourager le développement de cette action. Cet ultime vœu a été retenu pour l'édition 2018 (5). Si le soutien est toujours le bienvenu, notons cependant que cette action est simple à réaliser et très peu coûteuse (bien souvent le budget se réduit au coût du déplacement et de l'envoi postal). Elle permet donc d'entrevoir des modes d'actions culturelles soutenables, où seule la volonté et l'envie suffisent de part et d'autre pour 
enclencher le partage. Si l'action n'était pas connue dans les Hauts-de-France en 2015, elle l'est désormais, au vu de la production de films pour l'édition 2017 et des enthousiasmes partagés. Ce n'est pas la moindre des bonnes nouvelles, que ce soit le désir qui soit le maître mot.

\section{BIBLIOGRAPHIE}

Les films primés : www.museumexperts.com/sitem/musees_em_portables/presentation

D’autres films en ligne : www.formation-exposition-musee.fr/index.php?page=p\&p=100

Les films produits par le master expographie-muséographie : www.pearltrees.com/schaum

\section{RÉSUMÉS}

L'opération "Musées(em)portables" a pour objectif de faire réaliser par des lycéens des films de fiction au musée avec un téléphone portable : l'une des responsables du projet montre comment celui-ci permet au musée de renouer des liens avec un public réputé peu réceptif et transforme le regard porté par les jeunes sur le musée ainsi désacralisé, qui devient un lieu propice au développement de leur imaginaire.

\section{INDEX}

Mots-clés : musée, lycéens, film

\section{AUTEUR}

\section{ISABELLE ROUSSEL-GILLET}

accompagnatrice de projets au musée à l'université d'Artois et co-responsable du master expographie muséographie

isabelle.rousselgillet@univ-artois.fr 